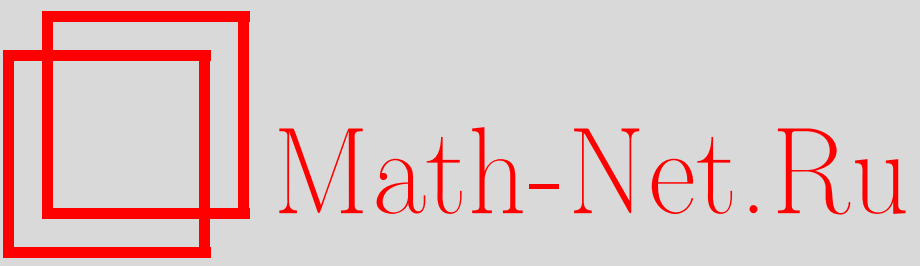

Ж. И. Абдуллаев, С. Н. Лакаев, Асимптотика дискретного спектра разностного трехчастичного оператора Шредингера на решетке, ТМФ, 2003, том 136, номер 2, 231-245

DOI: https://doi.org/10.4213/tmf218

Использование Общероссийского математического портала Math-Net.Ru подразумевает, что вы прочитали и согласны с пользовательским соглашением

http: //www . mathnet.ru/rus/agreement

Параметры загрузки:

IP : 54.198 .67 .100

26 апреля 2023 г., 14:43:03 
ТЕОРЕТИЧЕСКАЯ

И МАТЕМАТИЧЕСКАЯ

ФИЗИКА

Том 136, № 2

август, 2003

(C) 2003 г.

Ж.И. Абдуллаев*, С.Н. Лакаев*

\section{АСИМПТОТИКА ДИСКРЕТНОГО СПЕКТРА РАЗНОСТНОГО ТРЕХЧАСТИЧНОГО ОПЕРАТОРА ШРЕДИНГЕРА НА РЕШЕТКЕ}

На трехмерной целочисленной решетке рассматривается гамильтониан $H_{\mu}(K)$ системы трех бозонов, взаимодействующих с помощью парных контактных потенциалов притяжения. Получена асимптотика для числа $N(K, z)$ собственных значений, лежащих ниже $z \leqslant 0$ оператора $H_{\mu_{0}}(K)$ по полному квазиимпульсу $K \rightarrow 0$ и спектральному параметру $z \rightarrow-0$.

Ключевые слова: оператор Шредингера, существенный спектр, дискретный спектр, оператор Гильберта-Шмидта, асимптотика.

\section{1. ВВЕДЕНИЕ}

Одним из замечательных результатов спектрального анализа трехчастичного непрерывного оператора Шредингера является так называемый эффект Ефимова [1]: если в системе трех частиц, взаимодействуюших с помошњю парных короткодействуюших потенциалов, ни одна из трех двухчастичных подсистем не имеет связанных состояний с отрицательной энергией, но по меньшей мере две из них имеют резонанс с энергией в нуле, то у этой трехчастичной системы сушествует бесконечное число трехчастичных связанных состояний с отрицательной энергией, накапливающихся к нулю. Этот эффект описан в литературе (см. [2], [3]). Строгое математическое доказательство существования эффекта Ефимова было приведено впервые в работе [4], а затем в работах [5]-[7].

В моделях физики твердого тела [8] и решетчатой теории поля [9] возникают дискретные операторы Шредингера, являющиеся решетчатым аналогом обычного трехчастичного оператора Шредингера в непрерывном пространстве. Для таких операторов также естественно ожидать появления эффекта Ефимова, поскольку, как показывает тшательньй анализ этого эффекта [4], за него ответственна “длинноволновая часть” спектра оператора Шредингера, одинаковая в обоих случаях - решетчатом и непрерывном. На физическом уровне это было показано в работах [8], [10].

Хотя дискретный оператор Шредингера системы трех решетчатых частиц ограничен и возмушение в парной задаче - компактный оператор, спектр оператора Шредин-

* Самаркандский государственный университет, Самарканд, Узбекистан. E-mail: info@samarkand.uz,slakaev@mail.ru 
гера для системы трех квантовых решетчатых частиц имеет более сложный характер по сравнению с непрерывным случаем.

В непрерывном случае “двухчастичная" и “трехчастичная" ветви существенного спектра состоят из полуосей $[\kappa, \infty)$, где $\kappa \leqslant 0$, и пересекаются. Для дискретного оператора Шредингера “двухчастичная” и “трехчастичная” ветви существенного спектра состоят из отрезков конечной длины, причем они могут не пересекаться. Поэтому возникает вопрос: сушествуют ли собственные значения в лакунах и, если сушествуют, то конечно ли их число?

В работе [11] рассмотрен трехчастичный дискретный оператор Шредингера $H_{\mu}(K)$ ( $\mu>0$ - энергия взаимодействия двух частиц, $K \in T^{3}$ - полный квазиимпульс системы, $T^{3}$ - трехмерный тор), описывающий систему трех одинаковых частиц (бозонов), взаимодействующих с помощью парных контактных потенциалов притяжения, при общих предположениях на энергию отдельного бозона $\varepsilon(p), p \in T^{3}$, и доказано существование эфффекта Ефимова.

В работе [12] рассмотрен разностный трехчастичный дискретный оператор Шредингера, т.е. случай $\varepsilon(p)=\sum_{i=1}^{3}\left(1-\cos p_{i}\right)$. Показано, что при нулевом значении полного квазиимпульса $(K=0)$ и некотором $\mu=\mu_{0}>0$ имеет место эффект Ефимова, а при всех $K \in U_{\delta}^{o}(0)=\left\{p \in T^{3}: 0<|p|<\delta\right\}$ и $\mu>0$ оператор $H_{\mu}(K)$ имеет лишш конечное число собственных значений, лежащих вне существенного спектра.

Пусть $N(z), z<0,-$ число собственных значений трехчастичного непрерывного оператора Шредингера, лежаших левее точки $z<0$. В работе [6] получена нижняя оценка

$$
\lim _{z \rightarrow-0} \inf \frac{N(z)}{|\ln | z||}=\mathcal{U}_{0}, \quad 0<\mathcal{U}_{0} .
$$

Этот результат согласуется с результатами работы [13], где установлена экспоненциальная асимптотика собственных значений, соответствуюших сферически-симметричным связанным состояниям. В работе [7] найдена асимптотика

$$
\lim _{z \rightarrow-0} \frac{N(z)}{|\ln | z||}=\mathcal{U}_{0}, \quad 0<\mathcal{U}_{0}<\infty,
$$

где число $\mathcal{U}_{0}$ не зависит от потенциалов взаимодействий, а зависит только от отношения масс частиц.

Обозначим через $\tau(K)$ нижнюю грань существенного спектра дискретного трехчастичного оператора $H_{\mu_{0}}(K), K \in T^{3}$, и через $N(K, z)$ - число собственных значений этого оператора, лежаших левее точки $z \leqslant \tau(K)$.

В данной работе для числа $N(0, z)$ собственных значений оператора $H_{\mu_{0}}(0)$, лежащих левее точки $z<0$, получена аналогичная (1.1) асимптотика

$$
\lim _{z \rightarrow-0} \frac{N(0, z)}{|\ln | z||}=\frac{\lambda_{0}}{2 \pi}
$$

где $\lambda_{0}$ - единственное положительное решение уравнения

$$
\lambda \sqrt{3} \operatorname{ch} \frac{\pi \lambda}{2}=8 \operatorname{sh} \frac{\pi \lambda}{6} .
$$


Доказана конечность числа $N(K, \tau(K))$ для любого $K \in U_{\delta}^{o}(0)$. Для числа отрицательных собственных значений оператора $H_{\mu_{0}}(K)$ установлена асимптотическая формула

$$
\lim _{|K| \rightarrow 0} \frac{N(K, 0)}{|\ln | K||}=\frac{\lambda_{0}}{\pi}
$$

которая характерна только для решетчатых систем и не имеет аналогов в непрерывном случае.

Кроме того, показано отсутствие собственных значений дискретного оператора Шредингера $H_{\mu}(0)$ при "малых взаимодействиях $\mu(\mu \ll 1)$ " и установлено, что при "больших взаимодействиях $\mu$ ( $\mu \gg 1)$ " оператор имеет единственное простое собственное значение ниже сушественного спектра.

\section{2. ПОСТАНОВКА ЗАДАЧИ И ФОРМУЛИРОВКА РЕЗУЛЬТАТОВ}

Пусть $\mathbb{Z}^{3}$ - трехмерная целочисленная решетка, $\ell_{2}^{\mathrm{s}}\left(\left(\mathbb{Z}^{3}\right)^{m}\right)$ - гильбертово пространство квадратично-суммируемых функций, определенных на $\left(\mathbb{Z}^{3}\right)^{m}$ и симметричных относительно перестановки любых двух переменных.

В координатном представлении разностные операторы Шредингера системы двух и системы трех одинаковых частищ (бозонов) с парными контактными потеншиалами взаимодействия на трехмерной решетке действуют соответственно в пространствах $\ell_{2}^{\mathrm{S}}\left(\left(\mathbb{Z}^{3}\right)^{2}\right)$ и $\ell_{2}^{\mathrm{S}}\left(\left(\mathbb{Z}^{3}\right)^{3}\right)$ по формулам

$$
\begin{aligned}
& \left(\tilde{h}_{\mu} \varphi\right)\left(n_{1}, n_{2}\right)=\frac{1}{2} \sum_{|s|=1}\left[2 \varphi\left(n_{1}, n_{2}\right)-\varphi\left(n_{1}+s, n_{2}\right)-\varphi\left(n_{1}, n_{2}+s\right)\right]-\mu \delta_{n_{1} n_{2}} \varphi\left(n_{1}, n_{2}\right), \\
& \left(\widetilde{H}_{\mu} \varphi\right)\left(n_{1}, n_{2}, n_{3}\right)=\frac{1}{2} \sum_{|s|=1}\left[3 \varphi\left(n_{1}, n_{2}, n_{3}\right)-\varphi\left(n_{1}+s, n_{2}, n_{3}\right)-\right. \\
& \left.-\varphi\left(n_{1}, n_{2}+s, n_{3}\right)-\varphi\left(n_{1}, n_{2}, n_{3}+s\right)\right]- \\
& -\mu\left(\delta_{n_{1} n_{2}}+\delta_{n_{1} n_{3}}+\delta_{n_{3} n_{2}}\right) \varphi\left(n_{1}, n_{2}, n_{3}\right) .
\end{aligned}
$$

Здесь $\mu>0$ - энергия взаимодействия двух частищ, $\delta_{n m}$ - символ Кронекера.

Пусть $T^{3}$ - трехмерньй тор, $L_{2}^{\mathrm{s}}\left(\left(T^{3}\right)^{m}\right)$ - гильбертово пространство квадратичноинтегрируемых функций, определенных на $\left(T^{3}\right)^{m}$ и симметричных относительно перестановки любых двух переменных. В импульсном представлении операторы Шредингера систем двух и трех одинаковых частищ действуют соответственно в гильбертовых пространствах $L_{2}^{\mathrm{S}}\left(\left(T^{3}\right)^{2}\right)$ и $L_{2}^{\mathrm{S}}\left(\left(T^{3}\right)^{3}\right)$ по формулам

$$
\begin{aligned}
\left(h_{\mu} f\right)\left(k_{1}, k_{2}\right)= & \left(\varepsilon\left(k_{1}\right)+\varepsilon\left(k_{2}\right)\right) f\left(k_{1}, k_{2}\right)-\mu \int \delta\left(k_{1}+k_{2}-k_{1}^{\prime}-k_{2}^{\prime}\right) f\left(k_{1}^{\prime}, k_{2}^{\prime}\right) d k_{1}^{\prime} d k_{2}^{\prime}, \\
\left(H_{\mu} f\right)\left(k_{1}, k_{2}, k_{3}\right)= & \left(\varepsilon\left(k_{1}\right)+\varepsilon\left(k_{2}\right)+\varepsilon\left(k_{3}\right)\right) f\left(k_{1}, k_{2}, k_{3}\right)- \\
& -\mu \sum_{\alpha=1}^{3} \int \delta\left(k_{\alpha}-k_{\alpha}^{\prime}\right) \delta\left(k_{\beta}+k_{\gamma}-k_{\beta}^{\prime}-k_{\gamma}^{\prime}\right) f\left(k_{1}^{\prime}, k_{2}^{\prime}, k_{3}^{\prime}\right) d k_{1}^{\prime} d k_{2}^{\prime} d k_{3}^{\prime} .
\end{aligned}
$$

Здесь $\{\alpha, \beta, \gamma\}=\{1,2,3\}$,

$$
\varepsilon(p)=\sum_{i=1}^{3}\left(1-\cos p_{i}\right), \quad p=\left(p_{1}, p_{2}, p_{3}\right) \in T^{3},
$$


$\delta(k)$ - трехмерная дельта-функция Дирака. Знак интеграла без указания пределов интегрирования обозначает интеграл по всей области изменения переменных.

Пусть $k=k_{1}+k_{2}$ и $K=k_{1}+k_{2}+k_{3}$-полные квазиимпульсы системы двух и системы трех частиц, a

$$
\begin{aligned}
\gamma_{k} & =\left\{\left(k_{1}, k_{2}\right) \in\left(T^{3}\right)^{2}: k=k_{1}+k_{2}\right\}, \\
\Gamma_{K} & =\left\{\left(k_{1}, k_{2}, k_{3}\right) \in\left(T^{3}\right)^{3}: K=k_{1}+k_{2}+k_{3}\right\}
\end{aligned}
$$

- соответственно трехмерное и шестимерное многообразия. Обозначим через $L_{2}^{\mathrm{e}}\left(T^{3}\right) \subset$ $L_{2}\left(T^{3}\right)$ подпространство четных функций, а через $L(K)$ - гильбертово пространство всех квадратично-интегрируемых функций, определенных на $\left(T^{3}\right)^{2}$ и удовлетворяюших условиям $f\left(k_{1}, k_{2}\right)=f\left(k_{2}, k_{1}\right)=f\left(k_{1}, K-k_{1}-k_{2}\right)$.

Операторы $h_{\mu}$ и $H_{\mu}$ разлагаются в прямые операторные интегралы [11]

$$
h_{\mu}=\int \bigoplus h_{\mu}(k) d k, \quad H_{\mu}=\int \bigoplus H_{\mu}(K) d K,
$$

где ограниченные самосопряженные операторы $h_{\mu}(k), k \in T^{3}$, и $H_{\mu}(K), K \in T^{3}$, действуют в гильбертовых пространствах $L_{2}^{\mathrm{e}}\left(T^{3}\right)$ и $L(K)$, соответственно, по формулам

$$
\begin{gathered}
h_{\mu}(k) f(p)=\varepsilon_{k}(p) f(p)-\mu \int f(s) d s \\
H_{\mu}(K) f(p, q)=\varepsilon_{K}(p, q) f(p, q)-\mu \int[f(p, s)+f(s, q)+f(K-p-q, s)] d s .
\end{gathered}
$$

Здесь $\varepsilon_{k}(p)=\varepsilon(k / 2-p)+\varepsilon(k / 2+p)$ и $\varepsilon_{K}(p, q)=\varepsilon(K / 3+p)+\varepsilon(K / 3+q)+\varepsilon(K / 3-p-q)$. Пусть $\tau_{\mu}(K)$ - нижняя грань сушественного спектра $\sigma_{\text {ess }}\left(H_{\mu}(K)\right)$ оператора $H_{\mu}(K)$ и $N_{\mu}(K, z)$ - число собственных значений оператора $H_{\mu}(K)$, лежащих левее точки $z \leqslant$ $\tau_{\mu}(K)$. Положим

$$
\mu_{0}^{-1}=\frac{1}{2} \int \frac{d q}{\varepsilon(q)} .
$$

Для удобства воспользуемся обозначениями

$$
N(K, z)=N_{\mu_{0}}(K, z), \quad \tau(K)=\tau_{\mu_{0}}(K) .
$$

Сформулируем основные результаты работы.

Tеорема 2.1. Cуществует число $m, 0<m<\mu_{0}$, такое, что при всех $\mu \leqslant m$ оператор $H_{\mu}(0)$ не имеет собственных значений вне существенного спектра.

ТеОрема 2.2. Пусть $\mu=\mu_{0}$. Тогда оператор $H_{\mu_{0}}(0)$ имеет бесконечное число отрицательных собственньх значений, накапливающихся $к$ нулю, и для функций $N(0, z)$ и $N(K, 0)$ выполняются соотношения

$$
\lim _{z \rightarrow-0} \frac{2 N(0, z)}{|\ln | z||}=\lim _{|K| \rightarrow 0} \frac{N(K, 0)}{|\ln | K||}=\frac{\lambda_{0}}{\pi},
$$

где $\lambda_{0}$ - единственное положительное решение уравнения (1.2).

Tеорема 2.3. Существует число $M>0$ mакое, что при всех $\mu>M$ оператор $H_{\mu}(0)$ имеет единственное простое собственное значение $E(\mu, 0)$ левее существенного спектра, причем

$$
\lim _{\mu \rightarrow \infty} \frac{E(\mu, 0)}{\mu}=-3
$$




\section{3. СПЕКТР ДВУХЧАСТИЧНОГО ОПЕРАТОРА}

В соответствии с теоремой Вейля о сушественном спектре [14] непрерывный спектр $\sigma_{\mathrm{c}}\left(h_{\mu}(k)\right)$ оператора $h_{\mu}(k)$ совпадает со спектром $\sigma\left(h_{0}(k)\right)$ невозмушенного оператора $h_{0}(k)$, т.е. с отрезком $[m(k), M(k)]$, где

$$
m(k)=\min _{p \in T^{3}} \varepsilon_{k}(p), \quad M(k)=\max _{p \in T^{3}} \varepsilon_{k}(p) .
$$

Заметим, что при $k=(\pi, \pi, \pi)$ выполняется тождество $\varepsilon_{k}(q) \equiv 6$ и поэтому отрезок $[m(k), M(k)]$ преврашается в точку $\{6\}$.

Для функции $\varepsilon(p)$ имеет место представление $(2.1)$, поэтому $m(k)=2 \varepsilon(k / 2), M(k)=$ $12-2 \varepsilon(k / 2), k=\left(k_{1}, k_{2}, k_{3}\right) \in T^{3}$. Заметим, что эти функции $m(k)$ и $M(k)$ симметричны относительно перестановки любых двух переменных $k_{i}$ и $k_{j}$, четны по каждой переменной $k_{i} \in[-\pi, \pi], i=1,2,3$. Функция $m(k)$ строго возрастает, а $M(k)$ строго убывает по каждой переменной $k_{i} \in[0, \pi], i=1,2,3$. Следовательно, $\min _{k} m(k)=m(0)=0$, $\max _{k} M(k)=M(0)=12$.

Пусть $\Delta(\mu, k, z)$ - определитель Фредгольма оператора $I-\mu v r_{0}(k, z), r_{0}(k, z)$ - peзольвента оператора $h_{0}(k), v$-интегральньй оператор с ядром $v\left(q, q^{\prime}\right)=1$, т.е.

$$
v f=\int f(s) d s .
$$

Функция $\Delta(\mu, k, z)$ имеет вид

$$
\Delta(\mu, k, z)=1-\mu D(k, z), \quad D(k, z)=\int \frac{d q}{\varepsilon_{k}(q)-z} .
$$

Функция $D(k, \cdot)$ непрерывна и строго возрастает на каждой из полуосей $(-\infty, m(k))$ и $(M(k), \infty)$. Непрерывность функции $D(k, \cdot)$ следует из непрерывности подынтегральной функции, а монотонность вытекает из неравенства $D_{z}^{\prime}(k, z)>0$.

Из монотонности функции $D(k, \cdot)$ следует сушествование конечного или бесконечного предела

$$
\lim _{z \rightarrow m(k)} D(k, z)=D(k, m(k)) .
$$

Если для любого $i=1,2,3$ выполнено условие $k_{i} \neq \pi$, то этот предел конечен.

Лемма 3.1. Число $z \in C \backslash[m(k), M(k)]$ является собственным значением оператора $h_{\mu}(k)$ тогда и только тогда, когда $\Delta(\mu, k, z)=0$.

Доказательство можно найти в работе [11].

ОПРЕДЕЛЕНИЕ [4]. Будем говорить, что нижняя грань непрерывного спектра является виртуальным уровнем для оператора $h_{\mu}(0)$, если $\lambda=1$ является простым собственным значением оператора $\mu v r_{0}(0,0)$, причем для нетривиального решения $g=$ $\mu v r_{0}(0,0) g$ в пространстве $C\left(T^{3}\right)$ выполняется условие $g(0) \neq 0$.

Отметим, что требование наличия собственного значения $\lambda=1$ соответствует существованию решения уравнения Шредингера $h_{\mu}(0) f=0$, а из условия $g(0) \neq 0$ следует, что решение $f$ уравнения Шредингера не принадлежит пространству $L_{2}\left(T^{3}\right)$. 
Функция $D(k, m(k)), k=\left(k_{1}, k_{2}, k_{3}\right)$, симметрична относительно перестановки любых двух переменных $k_{i}, k_{j}$, четна по каж дой переменной $k_{i} \in[-\pi, \pi]$ и строго возрастает по $k_{i} \in[0, \pi], i=1,2,3$. При каждом фиксированном $z \leqslant 0$ функция $D(\cdot, z)$ строго убывает по каждой переменной $k_{i} \in[0, \pi], i=1,2,3$. При этом имеют место равенства

$$
\begin{aligned}
\min _{k \in T^{3}} D(k, m(k)) & =\max _{k} D(k, 0)=D(0,0)=\frac{1}{2} \int \frac{d q}{\varepsilon(q)}=\mu_{0}^{-1}, \\
\min _{k \in T^{3}} D(k, 0) & =D(\boldsymbol{\pi}, 0)=\frac{1}{6}, \quad \pi=(\pi, \pi, \pi) .
\end{aligned}
$$

Доказательство этих утверждений следует из свойств косинуса и монотонности интеграла Лебега (см. также [12]).

Лемма 3.2. Пусть $\mu=\mu_{0}$. Тогда $z=0$ является виртуальным уровнем для оператора $h_{\mu}(0)$, а при всех $k \neq 0$ оператор $h_{\mu}(k)$ имеет единственное простое положительное собственное значение $z_{\mu}(k)$ левее непрерывного спектра.

ДокАЗАТЕльСтво. В этом случае уравнение $\mu_{0} v r_{0}(0,0) g=g$ имеет решение $g(p) \equiv 1$, а уравнение Шредингера $h_{\mu}(0) f(p)=0$ имеет нетривиальное решение $f(p)=$ $(\varepsilon(p))^{-1}$, и это решение не принадлежит пространству $L_{2}\left(T^{3}\right)$, т.е. число 0 является виртуальным уровнем (не является собственным значением) оператора $h_{\mu}(0)$. Функция $D(k, m(k))$ строго возрастает, а $D(k, 0)$ строго убывает по $k_{i} \in[0, \pi], i=1,2,3$, поэтому при всех $k \neq 0$ имеем $\mu_{0} D(k, 0)<\mu_{0} D(0,0)=1<\mu_{0} D(k, m(k))$. Отсюда следует, что функция $\Delta\left(\mu_{0}, k, z\right)$ имеет единственный простой нуль в интервале $(0, m(k))$. Теперь из леммы 3.1 заключаем, что оператор $h_{\mu}(k)$ имеет единственное простое собственное значение $z_{\mu}(k)$, причем $0<z_{\mu}(k)<m(k)$.

Лемма 3.3. Собственное значение $z_{\mu}(k)=z_{\mu}\left(k_{1}, k_{2}, k_{3}\right)$ симметрично и четно по каждой переменной $k_{i} \in[-\pi, \pi], \quad i=1,2,3$, и строго возрастает по $k_{i} \in[0, \pi]$, $i=1,2,3$. При әтом

$$
z_{\mu_{0}}(0)=0, \quad z_{\mu}(\pi)=6-\mu .
$$

ДОКАЗАТЕЛЬСтво. В силу леммы 3.1 собственное значение $z_{\mu}\left(k_{1}, k_{2}, k_{3}\right)$ есть решение уравнения $\mu D(k, z)=1$. Симметричность и четность $z_{\mu}(k)$ следует из симметричности и четности функции $D(k, z)$. Докажем возрастание функции $z_{\mu}\left(k_{1}, k_{2}, k_{3}\right)$ по $k_{1}$ на отрезке $[0, \pi]$. Пусть $k_{1}^{\prime}<k_{1}^{\prime \prime} \in[0, \pi]-$ произвольные числа и $\mu D\left(k^{\prime}, z^{\prime}\right)=\mu D\left(k^{\prime \prime}, z^{\prime \prime}\right)=1$, где $k^{\prime}=\left(k_{1}^{\prime}, k_{2}, k_{3}\right), \quad k^{\prime \prime}=\left(k_{1}^{\prime \prime}, k_{2}, k_{3}\right)$. Функция $D(\cdot, z)$ строго убывает, поэтому $D\left(k^{\prime \prime}, z^{\prime}\right)<D\left(k^{\prime}, z^{\prime}\right)=D\left(k^{\prime \prime}, z^{\prime \prime}\right)$. Так как функция $D(k, \cdot)$ строго возрастает, то из условия $D\left(k^{\prime \prime}, z^{\prime}\right)<D\left(k^{\prime \prime}, z^{\prime \prime}\right)$ следует, что $z^{\prime}<z^{\prime \prime}$, т.е. $z\left(k^{\prime}\right)<z\left(k^{\prime \prime}\right)$. Теперь из соотношений $(3.1)$ и (3.2) следует (3.3). Лемма доказана.

Лемма 3.4. Собственное значение $z(\mu, k)=z_{\mu}(k)$ оператора $h_{\mu}(k)$ как функиия от $\mu$ строго убъвает на интервале $(0, \infty)$ и удовлетворяет неравенствам

$$
-\mu \leqslant z_{\mu}(k) \leqslant 6-\mu .
$$


ДокАЗАТЕЛЬСтво. В силу леммы 3.1 собственное значение $z(\mu, k)$ есть решение уравнения $\mu D(k, z)=1$. Пусть $\mu_{1}, \mu_{2} \in(0, \infty), \mu_{1}<\mu_{2}$, - два произвольных числа и $D\left(k, z_{1}\right)=1 / \mu_{1}>1 / \mu_{2}=D\left(k, z_{2}\right)$. Так как функция $D(k, \cdot)$ строго возрастает, то $z_{1}>z_{2}$. Это означает, что $z\left(\mu_{1}, k\right)>z\left(\mu_{2}, k\right)$. Из условия минимальности собственного значения самосопряженного оператора следует, что $-\mu \leqslant \inf \left(h_{\mu}(k) f, f\right)=z_{\mu}(k)$. Из условия монотонности находим $\max _{k} z_{\mu}(k)=6-\mu$. Лемма доказана.

\section{4. СПЕКТР ТРЕХЧАСТИЧНОГО ОПЕРАТОРА}

Здесь мы опишем сушественный спектр трехчастичного оператора $H_{\mu}(K)$, а исследование собственных значений этого оператора сведем к изучению собственных значений компактного оператора $A_{\mu}(K, z)$. Обозначим $E_{\mu K}(q)=z_{\mu}(2 K / 3-q)+\varepsilon(q+K / 3)$. Как известно [12], существенный спектр оператора $H_{\mu}(K)$ состоит из объединения областей значений двух функций $E_{\mu K}(q)$ и $\varepsilon_{K}(p, q)$, т.е.

$$
\sigma_{\mathrm{ess}}\left(H_{\mu}(K)\right)=\left[E_{\min }(\mu, K), E_{\max }(\mu, K)\right] \cup\left[\varepsilon_{\min }(K), \varepsilon_{\max }(K)\right],
$$

где

$$
\begin{gathered}
\varepsilon_{\min }(K)=\min _{p, q} \varepsilon_{K}(p, q)=3 \varepsilon\left(\frac{K}{3}\right), \quad \varepsilon_{\max }(K)=\max _{p, q} \varepsilon_{K}(p, q), \\
E_{\min }(\mu, K)=\min _{q} E_{\mu K}(q), \quad E_{\max }(\mu, K)=\max _{q} E_{\mu K}(q) .
\end{gathered}
$$

Первая часть $\left[E_{\min }(\mu, K), E_{\max }(\mu, K)\right]$ называется “двухчастичной” ветвью сушественного спектра трехчастичного оператора $H_{\mu}(K)$ и обозначается $\sigma_{\text {two }}\left(H_{\mu}(K)\right)$. Вторая часть $\left[\varepsilon_{\min }(K), \varepsilon_{\max }(K)\right]$ совпадает со спектром невозмущенного трехчастичного оператора $H_{0}(K)$ и называется "трехчастичной" ветвью существенного спектра оператора $H_{\mu}(K)$. Она обозначается $\sigma_{\text {three }}\left(H_{\mu}(K)\right)$.

Рассмотрим самосопряженный оператор (решетчатый аналог оператора ФаддееваНьютона, см. также [11]) $A_{\mu}(K, z), z \leqslant \tau_{\mu}(K)=\min _{q} E_{\mu K}(q)$, действующий в $L_{2}\left(T^{3}\right)$ по формуле

$$
\begin{aligned}
A_{\mu}(K, z) g(p)= & \frac{2 \mu}{\sqrt{\Delta(\mu, 2 K / 3-p, z-\varepsilon(p+K / 3))}} \times \\
& \times \int \frac{g(s) d s}{\sqrt{\Delta(\mu, 2 K / 3-s, z-\varepsilon(s+K / 3))}\left(\varepsilon_{K}(p, s)-z\right)} .
\end{aligned}
$$

Лемма 4.1 [11]. Число $z \in C \backslash \sigma_{\mathrm{ess}}\left(H_{\mu}(K)\right)$ является собственным значением оператора $H_{\mu}(K)$ тогда и только тогда, когда $\lambda=1$ есть собственное значение оператора $A_{\mu}(K, z)$.

Для самосопряженного оператора $B$, не имеющего сушественного спектра правее точки $\lambda$, обозначим через $n(\lambda, B)$ число собственных значений оператора $B$, превышаюших $\lambda>0$

Лемма 4.2. Для любого $z<\tau_{\mu}(K)$ имеет место равенство

$$
N_{\mu}(K, z)=n\left(1, A_{\mu}(K, z)\right) .
$$


ДокАЗАТЕЛЬСтво. Сначала докажем равенство

$$
N_{\mu}(K, z)=n\left(1, \mu R_{0}^{1 / 2}(K, z) V R_{0}^{1 / 2}(K, z)\right), \quad V=V_{1}+V_{2}+V_{3} .
$$

Пусть для некоторой функции $f \in L_{2}\left(T^{3}\right)$ выполняется неравенство $\left(H_{\mu}(K) f, f\right)<$ $z(f, f)$, что равносильно

$$
\left(\left(H_{0}(K)-z\right) f, f\right)<(\mu V f, f) .
$$

Неравенство (4.3) эквивалентно неравенству

$$
(g, g)<\left(\mu R_{0}^{1 / 2}(K, z) V R_{0}^{1 / 2}(K, z) g, g\right), \quad g=\left(H_{0}(K)-z\right)^{1 / 2} f .
$$

Отсюда получаем

$$
N_{\mu}(K, z) \leqslant n\left(1, \mu R_{0}^{1 / 2}(K, z) V R_{0}^{1 / 2}(K, z)\right) .
$$

Из обратимости оператора $\left(H_{0}(K)-z\right)$ можно получить и обратное неравенство к $(4.4)$, что и доказывает (4.2). Рассмотрим уравнение для собственных значений оператора $\mu R_{0}^{1 / 2}(K, z) V R_{0}^{1 / 2}(K, z)$, т.е. уравнение

$$
\lambda g=\sum_{i=1}^{3} \mu R_{0}^{1 / 2}(K, z) V_{i} R_{0}^{1 / 2}(K, z) g .
$$

Обозначая $\varphi(p)=\left(V_{i}^{1 / 2} R_{0}^{1 / 2}(K, z) g\right)(p)$ и учитывая равенство $V_{i}^{1 / 2}=V_{i}$, мы получим эквивалентное (4.5) уравнение

$$
\begin{aligned}
\lambda \varphi(p) & =\mu \int \frac{\varphi(p) d q}{\varepsilon_{K}(p, q)-z}+2 \mu \int \frac{\varphi(q) d q}{\varepsilon_{K}(p, q)-z}= \\
& =\mu V_{1} R_{0}(K, z) V_{1} \varphi+2 \mu V_{1} R_{0}(K, z) V_{2} \varphi .
\end{aligned}
$$

Отсюда следует, что

$$
n\left(1, \mu R_{0}^{1 / 2}(K, z) V R_{0}^{1 / 2}(K, z)\right)=n\left(1, \mu V_{1} R_{0}(K, z) V_{1}+2 \mu V_{1} R_{0}(K, z) V_{2}\right) .
$$

Теперь воспользуемся вариационным методом:

$$
(\varphi, \varphi)<\left(\mu V_{1} R_{0}(K, z) V_{1} \varphi, \varphi\right)+\left(2 \mu V_{1} R_{0}(K, z) V_{2} \varphi, \varphi\right)
$$

или

$$
\left(\left(I-\mu V_{1} R_{0}(K, z) V_{1}\right) \varphi, \varphi\right)<\left(2 \mu V_{1} R_{0}(K, z) V_{2} \varphi, \varphi\right) .
$$

Обозначив $u=\left(I-\mu V_{1} R_{0}(K, z) V_{1}\right)^{1 / 2} \varphi$, получим $(u, u)<\left(A_{\mu}(K, z) u, u\right)$. Таким образом,

$$
n\left(1, \mu R_{0}^{1 / 2}(K, z) V R_{0}^{1 / 2}(K, z)\right) \leqslant n\left(1, A_{\mu}(K, z)\right) .
$$

Поменяв аргументы, получим обратное к (4.6) неравенство, что приводит к равенству

$$
n\left(1, \mu R_{0}^{1 / 2}(K, z) V R_{0}^{1 / 2}(K, z)\right)=n\left(1, A_{\mu}(K, z)\right) .
$$

Из соотношений (4.7) и (4.2) следует (4.1). Лемма доказана. 
Лемма 4.3. Существует число $\delta>0$ mакое, что при всех $k \in U_{\delta}(0)$ u $z \in$ $U_{\delta}(m(k))=\{z:|z-m(k)|<\delta\}$ функиия $\Delta(\mu, k, z)$ разлагается в ряд Пюизо

$$
\Delta(\mu, k, z)=1-\mu \sum_{n=0}^{\infty} c_{n}(k)(m(k)-z)^{n / 2},
$$

где $c_{n}(k), \quad n=0,1,2, \ldots,-$ четная, вещественно-аналитическая функция в $U_{\delta}(0)$.

ДокАЗАТЕльСтво. Представление (4.8) получено в работе [15] при каждом фиксированном $k \in U_{\delta}(0)$. Аналитичность функций $c_{0}(k)=D(k, m(k)), c_{n}(k), n=1,2, \ldots$, по $k$ мы можем получить, пользуясь леммой Морса с параметром. Лемма доказана.

4.1. Случай $\mu=\mu_{0}$. Пусть $K=0$. Тогда

$$
\begin{aligned}
\min _{q} E_{\mu 0}(q) & =\min _{q \in T^{3}}\left[z_{\mu}(q)+\varepsilon(q)\right]=z_{\mu}(0)=0, \\
\max _{q \in T^{3}} E_{\mu 0}(q) & =\max _{q \in T^{3}}\left[z_{\mu}(q)+\varepsilon(q)\right]=12-\mu, \\
\min _{p, q} \varepsilon_{0}(p, q) & =0, \quad \max _{p, q} \varepsilon_{0}(p, q)=\frac{27}{2}
\end{aligned}
$$

Поэтому

$$
\sigma_{\mathrm{ess}}\left(H_{\mu}(0)\right)=[0,12-\mu] \cup\left[0, \frac{27}{2}\right]=\left[0, \frac{27}{2}\right] .
$$

При всех $K \in T^{3}, K \neq 0$, имеют место неравенства

$$
\begin{gathered}
0<\varepsilon_{\min }(K)=\min _{p, q} \varepsilon_{K}(p, q)=3 \varepsilon\left(\frac{K}{3}\right) \leqslant \frac{9}{2}, \\
\max _{q} E_{\mu K}(q) \geqslant z_{\mu}(K-\pi)+\varepsilon(\pi) \geqslant 6 .
\end{gathered}
$$

Отсюда следует, что “двухчастичная" и “трехчастичная” ветви сушественного спектра пересекаются, т.е.

$$
\left[E_{\min }(\mu, K), E_{\max }(\mu, K)\right] \cup\left[\varepsilon_{\min }(K), \varepsilon_{\max }(K)\right]=\left[E_{\min }(\mu, K), \varepsilon_{\max }(K)\right]
$$

и при этом $E_{\min }(\mu, K)<\varepsilon_{\min }(K)$. Действительно,

$$
\min _{q} E_{\mu K}(q) \leqslant z_{\mu}\left(\frac{2 K}{3}\right)+\varepsilon\left(\frac{K}{3}\right)<m\left(\frac{2 K}{3}\right)+\varepsilon\left(\frac{K}{3}\right)=3 \varepsilon\left(\frac{K}{3}\right) .
$$

Лемма 4.4. Существует число $\delta>0$ такое, что для любого $K \in U_{\delta}^{o}(0)=\{p \in$ $\left.T^{3}: 0<|p|<\delta\right\}$ оператор $A(K, z) \equiv A_{\mu_{0}}(K, z), \quad z \leqslant \tau(K)$, является оператором Гильберта-Шмидта и непрерывен в равномерной операторной топологии. 
ДокАЗАТЕЛЬСтво. При любом $z<\tau(K)$ ядро оператора $A(K, z)$ непрерывно и поэтому квадратично-интегрируемо. Докажем квадратичную интегрируемость ядра оператора $A(K, z)$ при $z=\tau(K)$. Функция $\varepsilon_{K}(p, q)-\tau(K)$ ограничена снизу положительной величиной $\varepsilon_{\min }(K)-\tau(K)$, поэтому имеет место неравенство

$$
|A(p, q ; K, \tau(K))|^{2} \leqslant C(K) \frac{\Delta^{-1}(2 K / 3-p, z-\varepsilon(p+K / 3))}{\Delta(2 K / 3-q, z-\varepsilon(q+K / 3))},
$$

где $\Delta(p, z)=\Delta\left(\mu_{0}, p, z\right)$. При всех $K \in U_{\delta}^{o}(0)$ функция

$$
\Delta\left(\frac{2 K}{3}-q, \tau(K)-\varepsilon\left(q+\frac{K}{3}\right)\right)
$$

имеет невырожденный минимум в точке $P(K)[12]$, поэтому

$$
\left|\Delta\left(\frac{2 K}{3}-q, \tau(K)-\varepsilon\left(q+\frac{K}{3}\right)\right)\right| \geqslant C|p-P(K)|^{2} .
$$

Переходя в сферическую систему координат, можно показать, что ядро $A(p, q ; K, \tau(K))$ является квадратично-интегрируемым.

В силу теоремы Лебега о предельном переходе под знаком интеграла получим, что

$$
\int|A(p, q ; K, \tau(K))-A(p, q ; K, z)|^{2} d p d q \rightarrow 0 \text { при } z \rightarrow \tau(K),
$$

что и дает равномерную непрерывность оператора $A(K, z)$ в точке $z=\tau(K)$. Лемма доказана.

Лемма 4.5. Для любого $K \in U_{\delta}^{o}(0)$ оператор $H_{\mu}(K)$ имеет лишь конечное число собственных значений ниже существенного спектра.

ДокаЗАтЕльство. Допустим противное, т.е. оператор $H_{\mu}(K)$ имеет бесконечное число собственных значений $z_{n}$ таких, что $\lim _{n \rightarrow \infty} z_{n}=\tau(K)$. Пусть $f_{n} \in L(K)$ - собственная функция оператора $H_{\mu}(K)$, соответствуюшая собственному значению $z_{n}$, тогда функция

$$
g_{n}(p)=\sqrt{\Delta\left(\frac{2 K}{3}-p, z_{n}-\varepsilon\left(p+\frac{K}{3}\right)\right)} \int f_{n}(p, s) d s \in L_{2}\left(T^{3}\right)
$$

удовлетворяет симметризованному уравнению Фаддеева-Ньютона при $z=z_{n}$, т.е. $g_{n}(p)=A\left(K, z_{n}\right) g_{n}(p)$. Нормируем $g_{n}$ и покажем, что

$$
\left\|g_{n}\right\|=\left\|A\left(K, z_{n}\right) g_{n}\right\| \leqslant\left\|A(K, \tau(K)) g_{n}\right\|+\left\|\left[A\left(K, z_{n}\right)-A(K, \tau(K))\right] g_{n}\right\| \rightarrow 0
$$

при $n \rightarrow \infty$. Это следует из равномерной непрерывности $A(K, z)$ в точке $z=\tau(K)$ и компактности предельного оператора $A(K, \tau(K))$. Полученное противоречие доказывает лемму. 
Лемма 4.6. Для функиии $\Delta(2 K / 3-p, z-\varepsilon(K / 3+p))$ имеют место следующие представления:

$$
\begin{gathered}
\Delta(-p, z-\varepsilon(p))=2 \pi^{2} \mu_{0} \sqrt{\frac{3 p^{2}}{4}-z}+O\left(p^{2}\right)+O(|z|) \quad \text { npu } \quad p \rightarrow 0, \quad|z| \rightarrow-0, \\
\Delta\left(\frac{2 K}{3}-p,-\varepsilon\left(\frac{K}{3}+p\right)\right)=2 \pi^{2} \mu_{0} \sqrt{\frac{K^{2}}{6}+\frac{3 p^{2}}{4}}+O\left(p^{2}\right)+O\left(K^{2}\right)
\end{gathered}
$$

nрu $p \rightarrow 0, \quad K \rightarrow 0$.

ДокАЗАТЕЛЬСтво. Так как функция $D(k, m(k))$ имеет минимум в точке $k=0$, то

$$
\Delta(k, m(k))=1-\mu_{0} D(k, m(k))=O\left(k^{2}\right) \text { при } k \rightarrow 0 .
$$

Из леммы 4.3 следует, что

$$
\begin{aligned}
\Delta(-p, z-\varepsilon(p))= & -\mu_{0} D(-p, m(p))-\mu_{0} c_{1}(p) \sqrt{m(p)-(z-\varepsilon(p))}- \\
& -\mu_{0} \sum_{n=2}^{\infty} c_{n}(p)[m(p)-(z-\varepsilon(p))]^{n / 2}
\end{aligned}
$$

где

$$
m(p)+\varepsilon(p)=\frac{3 p^{2}}{4}+O\left(p^{4}\right) \text { при } p \rightarrow 0
$$

и

$$
c_{1}(k)=-\frac{2 \pi^{2} \mu_{0}}{\sqrt{\cos \left(k_{1} / 2\right) \cos \left(k_{2} / 2\right) \cos \left(k_{3} / 2\right)}} .
$$

Следовательно,

$$
c_{1}(k)=-2 \pi^{2} \mu_{0}+O\left(k^{2}\right) \text { при } k \rightarrow 0 .
$$

Поэтому справедливо первое представление в (4.9).

Из (4.10) получаем

$$
\Delta\left(\frac{2 K}{3}-p, m\left(\frac{2 K}{3}-p\right)\right)=\sum_{|\alpha|=2}^{\infty} c_{\alpha}\left(\frac{2 K}{3}-p\right)^{\alpha}
$$

и

$m\left(\frac{2 K}{3}-p\right)+\varepsilon\left(p+\frac{K}{3}\right)=2 \varepsilon\left(\frac{2 K / 3-p}{2}\right)+\varepsilon\left(p+\frac{K}{3}\right)=\frac{K^{2}}{6}+\frac{3 p^{2}}{4}+O\left(|K|^{3}\right)+O\left(|p|^{3}\right)$ при $K \rightarrow 0$ и $p \rightarrow 0$. Отсюда следует справедливость представления (4.9). Лемма доказана.

Заметим, что для функции $\varepsilon_{K}(p, q)$ имеет место представление

$$
\varepsilon_{K}(p, q)=\frac{K^{2}}{6}+p^{2}+(p, q)+q^{2}+O\left(|p|^{4}\right)+O\left(|q|^{4}\right)+O\left(|K|^{4}\right), \quad K, p, q \rightarrow 0 .
$$

Оператор $A_{\mathrm{S}}(K, z)$ называется сингулярной частью оператора $A(K, z)$ и действует в $L_{2}\left(T^{3}\right)$ по формуле

$$
A_{\mathrm{S}}(K, z) w(p)=\frac{1}{\pi^{2}} \int \frac{\chi(p) \chi(q)\left(K^{2} / 6+3 q^{2} / 4-z\right)^{-1 / 4} w(q) d q}{\left(K^{2} / 6+3 p^{2} / 4-z\right)^{1 / 4}\left(K^{2} / 6+p^{2}+(p, q)+q^{2}-z\right)},
$$

где $\chi(\cdot)$ - характеристическая функция единичного шара $B_{1}=\left\{p \in T^{3}:|p|<1\right\}$.

3 Теоретическая и математическая физика, т. 136, № 2, 2003 г. 
Лемма 4.7. Разность $A(0, z)-A_{\mathrm{s}}(0, z)$ (соответственно $\left.A(K, 0)-A_{\mathrm{s}}(K, 0)\right)$ является оператором Гильберта-Шмидта при $z \leqslant 0$ (соответственно $\left.K \in T^{3}\right)$.

ДОКАЗАТЕЛЬСТво. Ядро

$$
\begin{aligned}
A(p, q ; 0, z)-A_{\mathrm{s}}(p, q ; 0, z)= & \frac{2 \mu}{\sqrt{\Delta(-p, z-\varepsilon(p))}} \frac{\left(\varepsilon_{0}(p, q)-z\right)^{-1}}{\sqrt{\Delta(-q, z-\varepsilon(q))}}- \\
& -\frac{1}{\pi^{2}} \frac{\chi(p) \chi(q)\left(p^{2}+(p, q)+q^{2}-z\right)^{-1}}{\left(3 p^{2} / 4-z\right)^{1 / 4}\left(3 q^{2} / 4-z\right)^{1 / 4}}
\end{aligned}
$$

оператора $A(0, z)-A_{\mathrm{s}}(0, z), z \leqslant 0$, непрерывно при $p, q \notin B_{1}$, поэтому оно квадратично-интегрируемо по области $\left(T^{3}\right)^{2} \backslash\left(B_{1}\right)^{2}$. В силу леммы 4.6 ядро оператора $A(0, z)-$ $A_{\mathrm{s}}(0, z)$ допускает оценку

$$
\left|A(p, q ; 0, z)-A_{\mathrm{S}}(p, q ; 0, z)\right| \leqslant C\left(|p|^{-1 / 2}+|q|^{-1 / 2}\right)
$$

для всех $p \in B_{1}, q \notin B_{1}$ и для всех $p \notin B_{1}, q \in B_{1}$. В случае $(p, q) \in\left(B_{1}\right)^{2}$ имеет место неравенство

$$
\left|A(p, q ; 0, z)-A_{\mathrm{s}}(p, q ; 0, z)\right| \leqslant \frac{C|p|^{-1 / 2}}{p^{2}+(p, q)+q^{2}}+\frac{C|q|^{-1 / 2}}{p^{2}+(p, q)+q^{2}}
$$

Переходя в сферическую систему координат и пользуясь неравенствами (4.11), (4.12), можно доказать квадратичную интегрируемость ядра оператора $A(0, z)-A_{\mathrm{s}}(0, z)$, $z \leqslant 0$. Аналогично доказывается принадлежность классу Гильберта-Шмидта оператора $A(K, 0)-A_{\mathrm{s}}(K, 0), K \in T^{3}$. Лемма доказана.

Гильбертово пространство $L_{2}\left(B_{1}\right)$ является инвариантным подпространством относительно оператора $A_{\mathrm{s}}(K, z)$. Этот оператор действует в $L_{2}\left(B_{1}\right)$ по формуле

$$
A_{\mathrm{S}}(K, z) w(p)=\frac{1}{\pi^{2}} \int_{B_{1}} \frac{\left(K^{2} / 6+3 q^{2} / 4-z\right)^{-1 / 4} w(q) d q}{\left(K^{2} / 6+3 p^{2} / 4-z\right)^{1 / 4}\left(K^{2} / 6+p^{2}+(p, q)+q^{2}-z\right)} .
$$

Получим асимптотическую формулу для числа собственных значений $N(0, z)$ оператора $A_{\mathrm{S}}(0, z)$. Оператор $A_{\mathrm{s}}(0, z)$ унитарно-эквивалентен оператору $A_{\mathrm{S}}^{(1)}(0, z)$, действующему в $L_{2}\left(B_{r}\right), r=|z|^{-1 / 2}$, по формуле

$$
\left(A_{\mathrm{S}}^{(1)}(0, z) w\right)(p)=\frac{1}{\pi^{2}} \int_{B_{r}} \frac{w(q) d q}{\left(3 p^{2} / 4+1\right)^{1 / 4}\left(p^{2}+(p, q)+q^{2}+1\right)\left(3 q^{2} / 4+1\right)^{1 / 4}} .
$$

Эта эквивалентность осушествляется с помошью унитарного преобразования $U_{r}$ : $L_{2}\left(B_{1}\right) \rightarrow L_{2}\left(B_{r}\right)$,

$$
\left(U_{r} f\right)(p)=r^{-3 / 2} f\left(r^{-1} p\right) .
$$

Если мы заменим $\left(3 p^{2} / 4+1\right)^{-1 / 4},\left(3 q^{2} / 4+1\right)^{-1 / 4}$ и $q^{2}+(p, q)+p^{2}+1$ на $\left(3 p^{2} / 4\right)^{-1 / 4} \times$ $(1-\chi(p)),\left(3 q^{2} / 4\right)^{-1 / 4}(1-\chi(q))$ и $q^{2}+(p, q)+p^{2}$ соответственно, то получим оператор 
$A_{\mathrm{S}}^{(2)}(0, z)$, разность которого с $A_{\mathrm{s}}^{(1)}(0, z)$ будет оператором Гильберта-Шмидта и равномерно непрерывна в точке $z=0$. Пространство $L_{2}\left(B_{r} \backslash B_{1}\right)$ инвариантно относительно оператора $A_{\mathrm{s}}^{(2)}(0, z)$. Ядро этого интегрального оператора имеет вид

$$
\frac{2}{\sqrt{3} \pi^{2}} \frac{|p|^{-1 / 2}|q|^{-1 / 2}}{q^{2}+(p, q)+p^{2}}
$$

Этот оператор унитарно-эквивалентен интегральному оператору $\mathbf{S}_{R}, R=(1 / 2)|\ln | z||$, действуюшему в пространстве $L_{2}\left((0, R) \times \mathbf{S}^{2}\right)$ по формуле

$$
\left(\mathbf{S}_{R} \varphi\right)(r, w)=\frac{1}{2 \sqrt{3} \pi^{2}} \int_{\mathbf{S}^{2}} \int_{0}^{R} \frac{\varphi\left(r^{\prime}, w^{\prime}\right) d r^{\prime} d w^{\prime}}{\operatorname{ch}\left(r-r^{\prime}\right)+\left(w-w^{\prime}\right) / 2} .
$$

Эквивалентность осуществляется унитарным оператором $M: L_{2}\left(B_{r} \backslash B_{1}\right) \rightarrow L_{2}((0, R) \times$ $\left.\mathbf{S}^{2}\right)$, где

$$
(M f)(r, \omega)=e^{3 r / 2} f\left(e^{\omega} r\right), \quad r \in(0, R), \quad \omega \in \mathbf{S}^{2} .
$$

Из теоремы 4.1 и леммы 4.9 работы [7] вытекает

ТеОРема 4.1. Имеет место равенство

$$
\lim _{z \rightarrow-0} \frac{n(1, A(0, z))}{|\ln | z||}=\lim _{R \rightarrow \infty} \frac{n\left(1, \mathbf{S}_{R}\right)}{R}=\frac{\lambda_{0}}{2 \pi}
$$

где $\lambda_{0}$ - положительное решение уравнения (1.2).

Теперь получим аналогичные результаты относительно числа $N(K, 0)$. С помошью унитарного отображения $U_{\rho}: L_{2}\left(B_{1}\right) \rightarrow L_{2}\left(B_{\rho}\right)$, определенного формулой (4.13), можно показать, что оператор $A_{\mathrm{s}}(K, 0)$ унитарно-эквивалентен оператору $A_{\mathrm{S}}^{(1)}(K, 0)$, действуюшему в $L_{2}\left(B_{\rho}\right), \rho=|K|^{-1}$, по формуле

$$
\left(A_{\mathrm{s}}^{(1)}(K, 0) w\right)(p)=\frac{1}{\pi^{2}} \int_{B_{\rho}} \frac{w(q) d q}{\left(3 p^{2} / 4+1 / 6\right)^{1 / 4}\left(p^{2}+(p, q)+q^{2}+1 / 6\right)\left(3 q^{2} / 4+1 / 6\right)^{1 / 4}} .
$$

В пространстве $L_{2}\left(B_{\rho}\right)$ рассмотрим оператор $A_{\mathrm{s}}^{(2)}(K, 0)$ с ядром

$$
\frac{2}{\sqrt{3} \pi^{2}} \frac{|p|^{-1 / 2}|q|^{-1 / 2}}{q^{2}+(p, q)+p^{2}}(1-\chi(p))(1-\chi(q)) .
$$

Разность $A_{\mathrm{s}}^{(1)}(K, 0)-A_{\mathrm{s}}^{(2)}(K, 0)$ является оператором Гильберта-Шмидта и непрерывна в равномерной операторной топологии в точке $K=0$. Наконец, оператор $M A_{\mathrm{s}}^{(2)}(K, 0) M^{-1}=\mathbf{S}_{R^{\prime}}, \quad R^{\prime}=|\ln | K||$, действует в пространстве $L_{2}\left(\left(0, R^{\prime}\right) \times \mathbf{S}^{2}\right)$ по формуле (4.14), где оператор $M$ определяется формулой (4.15). Разница состоит в том, что теперь величина $R^{\prime}=|\ln | K||$ зависит от $K \in T^{3}, \quad K \neq 0$.

Из теоремы 4.1 вытекает

Теорема 4.2. Для функиии $n(1, A(K, 0))$ выполняется соотношение

$$
\lim _{|K| \rightarrow 0} \frac{N(K, 0)}{|\ln | K||}=\lim _{|K| \rightarrow 0} \frac{n(1, A(K, 0))}{|\ln | K||}=\lim _{R^{\prime} \rightarrow \infty} \frac{n\left(1, \mathbf{S}_{R^{\prime}}\right)}{R^{\prime}}=\frac{\lambda_{0}}{\pi} .
$$

Теперь доказательство теоремы 2.2 (см. раздел 2) следует из равенств (4.16), (4.17). 


\section{2. Случай малых $\mu \ll 1$.}

ДОКАЗАТЕЛЬСТВО ТЕОРЕМЫ 2.1. При всех $\mu \in\left(0, \mu_{0}\right)$ существенный спектр $\sigma_{\text {ess }}\left(H_{\mu}(0)\right)=[0,27 / 2]$. Норма оператора $A_{\mu}(0,0)=A(0,0)$ непрерывно зависит от $\mu>$ 0 и допускает оценку $\left\|A_{\mu}(0,0)\right\| \leqslant C \mu$, поэтому сушествует число $m \in\left(0, \mu_{0}\right)$ такое, что при всех $\mu \in(0, m]$ имеем $\left\|A_{\mu}(0,0)\right\|<1$. Отсюда и из леммы 4.2 следует, что оператор $H_{\mu}(0)$ не имеет отрицательных собственных значений. В работе [12] доказано, что оператор $H_{\mu}(K)$ не имеет собственных значений правее сушественного спектра при всех $\mu>0$ и $K \in T^{3}$.

\section{3. Случай больших $\mu \gg 1$.}

ДОКАЗАТЕЛЬСТВО ТЕОРЕМЫ 2.3. При $\mu>\mu_{0}$ левый край $\tau(\mu)$ сушественного спектра оператора $H_{\mu}(0)$ равен $z_{\mu}(0)$. В силу $(3.4)$ имеем $-\mu \leqslant \tau(\mu)$. Применяя вариационный метод, получаем

$$
-3 \mu \leqslant \inf _{\|f\|=1}\left(H_{\mu}(0) f, f\right) \leqslant 9-3 \mu .
$$

Отсюда следует, что при $\mu>0$ наименьшее собственное значение $E(\mu, 0)$ оператора $H_{\mu}(0)$ лежит в интервале $(-3 \mu, 9-3 \mu)$. Поэтому имеет место равенство (2.3). Теперь докажем, что оператор $H_{\mu}(0)$ имеет единственное простое собственное значение, лежащее ниже существенного спектра. В силу леммы 4.2 для этого достаточно показать, что оператор $A_{\mu}\left(0, z_{\mu}(0)\right)$ имеет единственное простое собственное значение, большее 1 . Оператор $A_{\mu}\left(0, z_{\mu}(0)\right)$ представим в виде суммы двух операторов

$$
A_{\mu}\left(0, z_{\mu}(0)\right)=A_{\mu}^{(0)}\left(0, z_{\mu}(0)\right)+A_{\mu}^{(1)}\left(0, z_{\mu}(0)\right)
$$

где

$$
\begin{aligned}
\left(A_{\mu}^{(0)}\left(0, z_{\mu}(0)\right) f\right)(p)= & -\frac{2 \mu}{z_{\mu}(0) \sqrt{\Delta\left(\mu,-p, z_{\mu}(0)-\varepsilon(p)\right)}} \int \frac{f(q) d q}{\sqrt{\Delta\left(\mu,-q, z_{\mu}(0)-\varepsilon(q)\right)}}, \\
\left(A_{\mu}^{(1)}\left(0, z_{\mu}(0)\right) f\right)(p)= & \frac{2 \mu}{\sqrt{\Delta\left(\mu,-p, z_{\mu}(0)-\varepsilon(p)\right)}} \times \\
& \times \int \frac{\varepsilon_{0}(p, q) f(q) d q}{z_{\mu}(0)\left(\varepsilon_{0}(p, q)-z_{\mu}(0)\right) \sqrt{\Delta\left(\mu,-q, z_{\mu}(0)-\varepsilon(q)\right)}} .
\end{aligned}
$$

Оператор $A_{\mu}^{(0)}\left(0, z_{\mu}(0)\right)$ одномерен и имеет собственное значение

$$
\lambda(\mu)=-\frac{2 \mu}{z_{\mu}(0)} \int \frac{d q}{\Delta\left(\mu,-q, z_{\mu}(0)-\varepsilon(q)\right)}>1,
$$

что следует из неравенств $0<\Delta\left(\mu,-q, z_{\mu}(0)-\varepsilon(q)\right)<1$ и $-\mu \leqslant z_{\mu}(0) \leqslant 6-\mu$. Норма оператора $A_{\mu}^{(1)}\left(0, z_{\mu}(0)\right)$ допускает оценку сверху

$$
\| A_{\mu}^{(1)}\left(0, z_{\mu}(0) \| \leqslant \frac{C}{\mu}\right.
$$

при больших $\mu \gg 1$. Из вышеизложенного вытекает, что $n\left(1, A_{\mu}\left(0, z_{\mu}(0)\right)=1\right.$. В силу леммы 4.4 получаем единственность собственного значения оператора $H_{\mu}(0)$, лежащего ниже $z_{\mu}(0)$. Теорема доказана. 
При $\mu>12$ “двухчастичная" ветвь $\sigma_{\text {two }}\left(H_{\mu}(0)\right)$ и “трехчастичная" ветвь $\sigma_{\text {three }}\left(H_{0}(0)\right)$ не пересекаются и появляется лакуна $(12-\mu, 0)$. Если $\mu>12$, то функция $\Delta(\mu,-p, z-\varepsilon(p))$ отрицательна при $z \in(12-\mu, 0)$. Поэтому симметризованный оператор имеет вид

$$
A_{\mu}(0, z) g(p)=-\frac{2 \mu}{\sqrt{-\Delta(\mu,-p, z-\varepsilon(p))}} \int \frac{\left(\varepsilon_{0}(p, s)-z\right)^{-1} g(s) d s}{\sqrt{-\Delta(\mu,-s, z-\varepsilon(s))}} .
$$

В случае $z=0$ и $z=12-\mu$ ядро оператора Фаддеева-Ньютона $A_{\mu}(0, z)$ имеет квадратично-интегрируемые особенности вида $\left(p^{2}+q^{2}\right)^{-1}$ и $\left(\sqrt{(p-\pi)^{2}} \sqrt{(q-\pi)^{2}}\right)^{-1}$, соответственно. Из компактности предельных операторов $A_{\mu}(0,0)$ и $A_{\mu}(0,12-\mu)$ вытекает следующая теорема (см. также теорему 2 в работе [16]).

ТЕОРема 4.3. Пусть $\mu>12$. Тогда оператор $H_{\mu}(0)$ имеет лишь конечное число собственных значений в лакуне $(12-\mu, 0)$.

Благодарности. Авторы выражают глубокую благодарность Р. А. Минлосу за внимание к работе и обсуждения полученных результатов. Работа финансировалась Фондом фундаментальных исследований РУз и Deutsche Forschungsgemeinschaft (грант № 436 USB $113 / 3)$.

\section{Список литературы}

[1] В. Н. Ефимов. ЯФ. 1970. Т. 12. № 5. С. 1080-1091.

[2] С. П. Меркурьев, Л. Д. Фаддеев. Квантовая теория рассеяния для систем нескольких частиц. М.: Наука, 1985.

[3] R. D. Amado, J. V. Noble. Phys. Rev. D. 1971. V. 5. № 8. P. 1992-2002.

[4] Д. Р. Яфаев. Матем. сб. 1974. Т. 9(136). № 4(8). С. 567-592.

[5] Yu. N. Ovchinnikov, I. M. Sigal. Ann. Phys. 1989. V. 123. P. 274-295.

[6] H. Tamura. J. Funct. Anal. 1991. V. 95. P. 433-459.

[7] A. V. Sobolev. Commun. Math. Phys. 1993. V. 156. P. 101-126.

[8] D. C. Mattis. Rev. Mod. Phys. 1986. V. 58. № 2. P. 361-379.

[9] В. А. Малышев, Р. А. Минлос. Тр. сем. им. И. Г. Петровского. 1983. № 9. С. 63-80.

[10] A. I. Mogilner. The problem of a few quasi-particles in solid-state physics. In: Applications of Self-Adjoint Extensions in Quantum Physics. Proc. Conf. (Dubna, USSR, 1987). Lect. Notes Phys. V. 324. Eds. P. Exner, P. Seba. Berlin: Springer, 1989. P. 160-173.

[11] С. Н. Лакаев. Функц. анализ и его прилож. 1993. Т. 27. № 3. С. 15-28.

[12] С. Н. Лакаев, ЖК. И. Абдуллаев. Матем. заметки. 2002. Т. 71. № 5. С. 686-696.

[13] S. Albeverio, R. Hoegh-Krohn, T. T. Wu. Phys. Lett. A. 1971. V. 83. P. 105-109.

[14] М. Рид, Б. Саймон. Методы современной математической физики. Т. 4. Анализ операторов. М.: Мир, 1982.

[15] С. Н. Лакаев. ТМФ. 1991. Т. 89. № 1. С. 94-104.

[16] Ж. И. Абдуллаев, С. Н. Лакаев. ТМф. 1997. Т. 111. № 1. С. 94-108.

Поступила в редакцию 23.VII.2002 г., после доработки 30.XI.2002 г. 